\title{
An Efficient Brain Image Classification Using Probabilistic Neural Network and Tumor Detection Using Image Processing
}

\author{
Kshitija V. Shingare ${ }^{1}$, N. D. Pergad ${ }^{2}$ \\ PG Student, E\&TC Department, STB College, Tuljapur, India ${ }^{1}$ \\ Assistant Professor, E\&TC Department, STB College, Tuljapur, India ${ }^{2}$
}

\begin{abstract}
The brain is the frontal part of the central nervous system. Brain tumor is an irregular growth caused by cells reproducing themselves in an uncontrolled manner. Brain tumor is may be serious and critical because of space formed inside the skull. So detection, diagnosis and evaluation of brain tumor are an important task in earlier stages. Brain tumor detection helps in finding the exact size and location of tumor. In this paper an efficient algorithm is proposed for brain tumor detection \& identification using image processing and classification is done using Probabilistic Neural Network Techniques. These techniques use the MRI Scanned Images to detect the tumor in the brain. Probabilistic Neural Network with radial basis function will be used to implement an automatic Brain Tumor classification. Decision making was performed in two stages: feature extraction using GLCM and the classification using PNN network. The performance of this classifier was calculated in terms of training performance and classification accuracies. The simulated results shown that classifier and segmentation algorithm provides better accuracy than previous methods.
\end{abstract}

Keywords: Brain Tumor; MRI; Probabilistic Neural Network; GLCM: Classifier; Segmentation;

\section{INTRODUCTION}

A tumor can be defined as a mass grows without any performance of the classifier. After classification the control. Generally treatments of Brain Tumor are partitioning is performed on the tomorous image for determined by:

- Age of Patient

- Medical history

- Type of Tumor

- Location and

- Size of Tumor [9]

Real time diagnosis of tumors by using more reliable algorithms has been the main focus of the latest developments in medical imaging and detection of brain tumor in MR images and CT scan images has been an active research area. The main problems faced by most of the medical imagery diagnosis systems are the separation of the cells and their nuclei from the rest of the image content. The process of separation i.e. segmentation is most important in the construction of a robust and effective diagnosis system. Images Segmentation is performed on the input images. This enables easier analysis of the image thereby leading to better tumor detection efficiency. Hence image segmentation is the fundamental problem in tumor detection. But before image segmentation a major stage in image processing is classification. A good classification process gives right decision and provides good and appropriate treatment. The classification of the given input image should be done under two classes' i.e. normal and abnormal class. Classification is done using the features of the tumor containing image and normal image. In feature extraction, the transformation of input image data into sets of features is done. If the accurate features are extracted from MRI then the further processing can be done quickly. Feature extraction plays a crucial role in determining the extracting the tumor region. The main contributions of our proposed technique are given as below:

- Pre-processing of the input brain MR image using Gaussian filter \& histogram equalization

- Feature extraction for the ROI is done with the help of Gray Level Co-occurrence Matrix (GLCM)

- Selected features are given as input to Probabilistic Neural Network (PNN) for training, and finally classify Normal (non-tumorous) image and Abnormal (tumorous) image

- Then the classified tumorous image is segmented out to locate tumor.

\section{A. Structure of Brain}

Generally human brain consists three main parts which controls different activities.

\section{Cerebrum}

The cerebrum controls learning, thinking, emotions, speech, reading, writing, problem solving etc.

\section{Cerebellum}

The cerebellum controls movement, standing, balance, \& complex actions

\section{Brain Stem}

Brain stem joints the brain with spinal cord. It controls blood pressure, body temperature \& breathing \& also controls some basic functions. [2] 


\section{B. Types of Tumour}

There are three general types of Tumour: 1. Benign

2. Pre-malignant 3. Malignant

1. Benign Tumour: A benign tumor is a tumour which does not expand in an abrupt way; it doesn't affect its neighbouring healthy tissues and also does not expand to non-adjacent tissues

2. Pre-Malignant Tumour: Premalignant Tumour is a precancerous stage. It is considered as a disease, if not properly treated it may lead to cancer.

3. Malignant Tumor: Malignancy is the type of tumour, which grows worst with the passage of time and ultimately results in the death of a person. The term malignant tumour is typically used for the description of cancer. [7]

\section{Diagnosis}

MRI (Magnetic Resonance Imaging) \& CT (Computed Tomography) are basically used in the biomedical to detect and visualize finer details in the internal structure of the body. CT uses ionizing radiation but MRI uses strong magnetic field to align the nuclear magnetization then radio frequencies changes the alignment of the magnetization which can be detected by the scanner. That signal can be further processed to create the extra information of the body.[7] MR image is safe as compared to CT scan image as it does not affect human body.[3]

Information about final paper submission is available from the conference website.

\section{Proposed Methodology}

The proposed algorithm starts by reading the input brain MR image and converting it into grey scale image. There are four major steps in the proposed approach. The first step is pre-processing; the second step is feature extraction using GLCM; the third step is classification using PNN; and last step is segmentation. Fig 1 gives sequence of the proposed technique.

\section{A. Image Acquisition}

Images are obtained using MRI scan \& displayed in 2D having pixels as its elements. MRI scan were stored in database of images in JPEG image formats. These images are displayed as a gray scale images. The entries of gray scale images are ranging from 0 to 255 , where o indicates total black colour and 255 represents total white colour. [3]

\section{B. Pre-Processing Stage}

The Contrast Limited Adaptive Histogram Equalization (CLAHE) is an enhanced version of adaptive histogram equalization. The contrast limited adaptive histogram equalization algorithm partitions the images into contextual regions and applies the histogram equalization to each region. These evens out the distribution of used gray values and by using this make unknown features of the image more visible. The amount of contrast enhancement for some intensity is directly proportional to the slope of the Cumulative Distribution Function (CDF) at that intensity level. Therefore by limiting the slope of the CDF, contrast enhancement can also be limited. The slope of CDF at a bin location is evaluated by the height of the histogram for that bin. Therefore the height limitation of the histogram limits the slope of the CDF and that's why the amount of contrast enhancement.

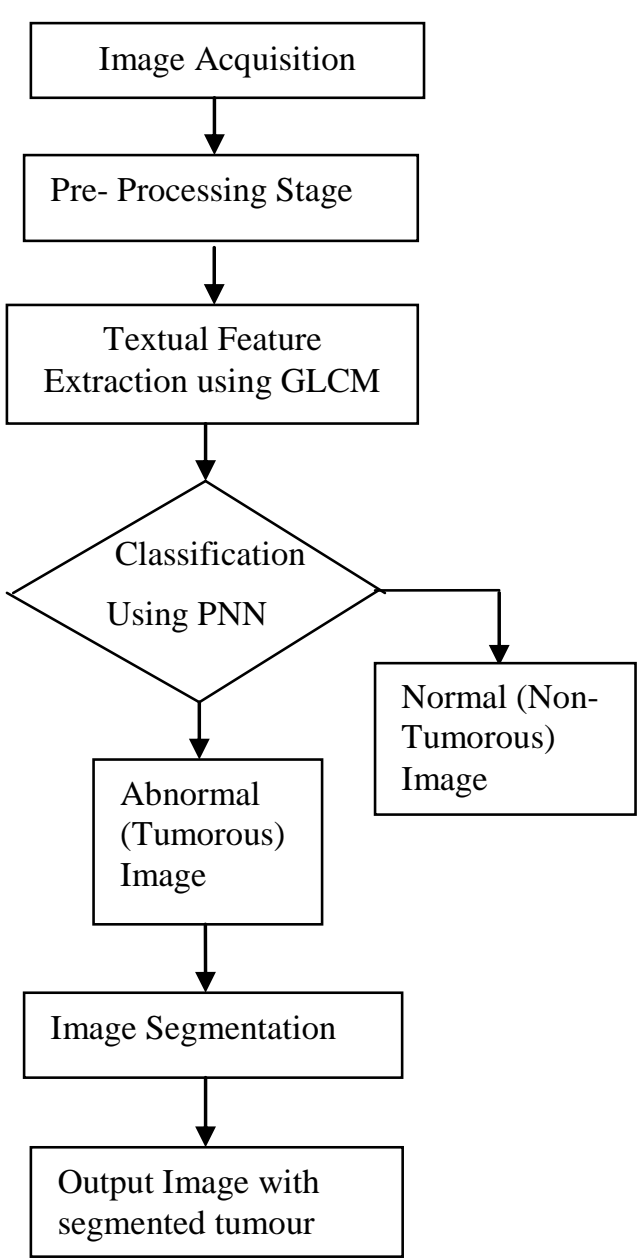

Fig 1 Flow chart of Proposed Methodology

\section{Extraction of Texture Feature}

Gray-level co-occurrence matrix (GLCM) is the statistical method of investigating the textures that considers the spatial relationship of the pixels. The GLCM functions characterize the texture of an image by calculating how frequently pairs of pixel with specific values and in a specified spatial relationship that present in an image, forms GLCM. This forms the extraction of statistical measures from this matrix. [10]

Here we are using Statistical approach to texture analysis among the four approaches (Structural, Statistical, model based and Transform). It is the most broadly used and more generally applied method because of its high accuracy and less computation time.

A gray level co-occurrence matrix (GLCM) contains information about the positions of pixels having similar gray level values. The GLCM, C, is defined with respect to 
given (row, column) displacement h. And element (i , j), denoted $\mathrm{c}_{\mathrm{ij}}$, is the number of times a point having gray level $\mathrm{j}$ occurs in position $\mathrm{h}$ relative to a point having gray Where level $\mathrm{i}$. Let $\mathrm{Nh}$ be the total number of pairs, then $\mathrm{C}_{\mathrm{ij}}=\mathrm{c}_{\mathrm{ij}}$ $\mathrm{Nh}$ is the elements of the normalized GLCM, C.

The probability measure can be defined as:

$$
P_{r(x)}=\left\{C_{i j}|\delta, \theta|\right\}
$$

Where $\mathrm{Cij}$ (the co-occurrence probability between grey levels $i$ and $j$ ) is defined as:

$$
C_{i j} \quad=\frac{P_{i j}}{\sum_{i, j=1}^{G} P_{i j}}
$$

Where Pij represents the number of occurrences of grey levels $\mathrm{i}$ and $\mathrm{j}$ within the given window, given a certain $(\delta$ inter pixel distance, $\theta$-orientation) pair. $\mathrm{G}$ is the quantized number of grey levels. The result of a texture calculation is a single number representing the entire window. This number is put in the place of the centre pixel of the window, then the window is moved one pixel and the process is repeated of calculating a new GLCM and a new texture measure. In this way an entire image is built up of texture values.

From the co-occurrence matrix obtained, we have to extract the 12 different statistical features. These are given as follows:

\section{Contrast}

Contrast is a measure of the local variations present in an image. It is given as,

$$
C(k, n)=\sum_{i} \sum_{j}(i-j)^{k} P d[i, j]^{n}
$$

If there is a large amount of variation in an image the $\mathrm{P}$ [i, j]'s will be concentrated away from the main diagonal and contrast will be high (typically $\mathrm{k}=2, \mathrm{n}=1$ ).

\section{Sum of Squares, Variance}

$V A R I A N C E=\sum_{I=0}^{G-1} \sum_{j=0}^{G-1}(i-\mu)^{2} P(i, j)$

This feature puts relatively high weights on the elements that differ from the average value of $P(i, j)$

\section{Correlation}

Correlation is a measure of image linearity

$$
C_{c}=\frac{\sum_{i} \sum_{j}\left[C_{i j} P d[i, j]\right]-\mu_{i \mu_{j}}}{\sigma_{i} \sigma j}
$$

$$
\mu_{i}=\sum i P_{d}[i, j] \sigma_{i}^{2}=\sum i^{2} P_{d}[i . j]-\mu_{i}^{2}
$$

\section{Energy}

One approach to generating texture features is to use local kernels to detect various types of texture. After the convolution with the specified kernel, the texture energy measure (TEM) is computed by summing the absolute values in a local neighborhood:

$$
L_{e}=\sum_{1}^{M} \sum_{1}^{N}|C(i, j)|
$$

If $\mathrm{n}$ kernels are applied, the result is an $\mathrm{n}$-dimensional feature vector at each pixel of the image being analyzed.

\section{Maximum Probability}

This is simply the largest entry in the matrix, and corresponds to the strongest response. This could be the maximum in any of the matrices or the maximum overall.

$$
C_{m}=\operatorname{MAX} \operatorname{Pd}[i, j]
$$

\section{Dissimilarity}

$$
\sum_{i, j=1}^{G} C i j|i-j|
$$

\section{Autocorrelation}

Other statistical approaches include an autocorrelation function, which has been used for analysing the regularity and coarseness of texture by Keizer. This function evaluates the linear spatial relationships between primitives. The set of autocorrelation coefficients shown below are used as texture features large value of MD indicates test image is of poor quality. Ideally it should be zero.

$$
\begin{aligned}
& C(p, q) \\
& =\frac{M N}{(M-p)(N-q)} \frac{\sum_{i=1}^{M-p} \sum_{j=1}^{N-q} f(i, j) f(i+p, j+q)}{\sum_{i=1}^{M} \sum_{j=1}^{N} f^{2}(i, j)}
\end{aligned}
$$

\section{Inverse different Moment}

IDM is also influenced by the homogeneity of the image. Because of the weighting factor IDM will get small contributions from inhomogeneous areas. The result is a low IDM value for inhomogeneous images, and a relatively higher value for homogeneous images. 
$I D M=\sum_{i=0}^{G-1} \sum_{j=0}^{G-1} \frac{1}{1+(i-j)^{2}} P(i, j)$

\section{Entropy}

Entropy is a measure of information content. It measures the randomness of intensity distribution.

$$
C_{e}=-\sum_{i} \sum_{j} P_{d}[i, j] \ln P_{d}[i, j]
$$

\section{Homogeneity}

A homogeneous image will result in a co-occurrence matrix with a combination of high and low $\mathrm{P}[\mathrm{i}, \mathrm{j}]$ 's.

$$
=\sum_{i} \sum_{j} \frac{P_{d}[i, j]}{1+|i-j|}
$$

\section{Cluster Prominence}

$$
\begin{aligned}
P R O M=\sum_{i=0}^{G-1} \sum_{j=0}^{G-1}\left\{i+j-\mu_{x}-\mu_{y}\right\}^{4} \\
\times P(i, j)
\end{aligned}
$$

12. Cluster Shade

$$
\begin{aligned}
& S H A D E=\sum_{I=0}^{2 G-2}(i \\
& -2 \mu)^{3} H_{s}(i \mid \Delta x, \Delta y)
\end{aligned}
$$

Where

$$
\mu=\frac{1}{2} \sum_{i=0}^{2 G-2} i H_{s}(i \mid \Delta x, \Delta y)
$$

\section{Classification by using Probabilistic Neural Network (PNN)}

A probabilistic neural network (PNN) is a feed forward neural network, resulting from the Bayesian network and a statistical algorithm called Kernel Fisher discriminate analysis. In a PNN, the operations are organized into a multilayered feed forward network with four layers as Input layer, Hidden layer, Pattern layer/Summation layer, Output layer.

\section{1) Architecture of probabilistic neural network}

In the early 1990s, Donald F. Specht proposed a method to formulate the weighted-neighbour method in the form of a neural network. He called this a "Probabilistic Neural Network". Fig 2 shown below gives a diagrammatic representation of a PNN network.
PNN is generally used in classification problems. When an input is present, the first layer computes the distance from the input vector to the training input vectors. This produces a vector whose elements indicate how close the input is to the training input. The second layer sums the contribution for each class of inputs and produces its total output as a vector of probabilities. Finally, a whole transfer function on the output of the second layer picks the maximum of these probabilities, and produces a 1 (positive identification) for that class and a 0 (negative identification) for non-targeted classes. [9]

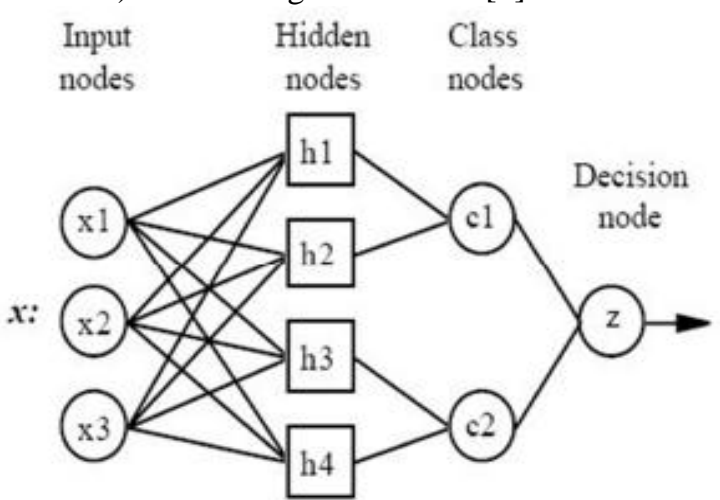

Fig 2 Architecture of Probabilistic Neural Network

\section{E. Image Segmentation}

Image segmentation is the process of partitioning a digital image into multiple segments (sets of pixels, which are also called as super pixels). The main aim of segmentation is to simplify or to change the representation of an image into something which is more meaningful \& easy to understand. [6]

In medical field image segmentation is typically used to study anatomical structure, to identify Region of interest (i.e. to locate tumour \& other abnormalities), used in treatment planning etc.[4] There are various techniques of image segmentation such as Thresholding, compression based methods, Region growing Techniques, Edge Detection Techniques, Clustering Methods, Watershed Segmentation etc. Here we detect tumour using edge detection \& basic morphology.

Step 1: Read the input brain MRI image.

Step 2: The gradient image can be calculated and a threshold can be applied to create a binary mask containing the segmented cell. First, we use edge and the canny operator to calculate the threshold value. We then tune the threshold value and use edge again to obtain a binary mask that contains the segmented cell.

Step 3: Dilate the Image. The binary gradient mask shows lines of high contrast in the image. These lines do not quite delineate the outline of the object of interest. Compared to the original image, you can see gaps in the lines surrounding the object in the gradient mask. These linear gaps will disappear if the canny image is dilated using linear structuring elements, which we can create with the strel function. The binary gradient mask is dilated using the vertical structuring element followed by the horizontal structuring element. The imdilate function dilates the image. 


\section{International Journal of Advanced Research in Computer and Communication Engineering}

Vol. 4, Issue 5, May2015

Step 4: Fill Interior Gaps. The dilated gradient mask shows the outline of the cell quite nicely, but there are still holes in the interior of the cell. To fill these holes we use the imfill function.

Step 5: In some applications, it is helpful to be able to separate out the regions of the image corresponding to objects in which we are interested, from the regions of the image that correspond to background. Thresholding is an easy and suitable method to achieve this binarization on the basis of the different intensities or colours in the foreground and background regions of an image. For the binarization of equalized image a thresholding technique is used as shown below:

Binarized Image bi, $\mathrm{j}=255$ if e $(\mathrm{i}, \mathrm{j})>\mathrm{T}$

Else bi, $\mathrm{j}=0$

Where e ( $\mathrm{i}, \mathrm{j})$ is the equalized MRI image and $\mathrm{T}$ is threshold resultant for the equalized image.[11]

\section{III.EXPERIMENTAL RESULTS}

Fig. 3 shows Brain MR Image as an input to the proposed system.
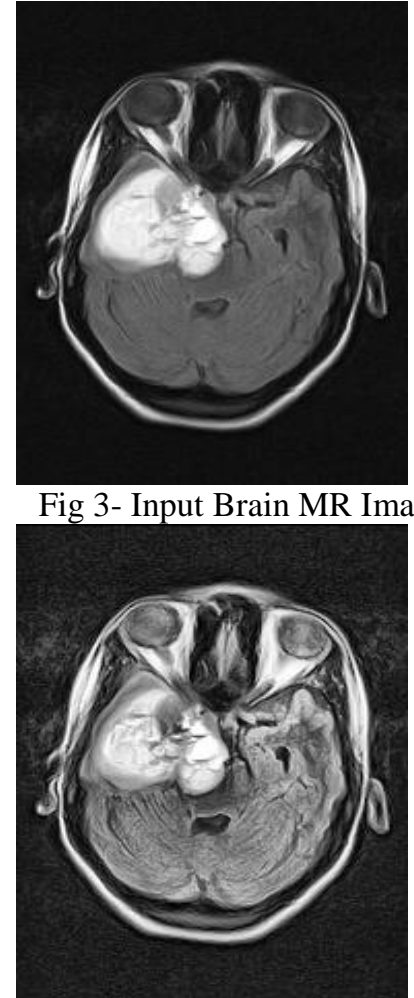

Fig 4- Filtered Image

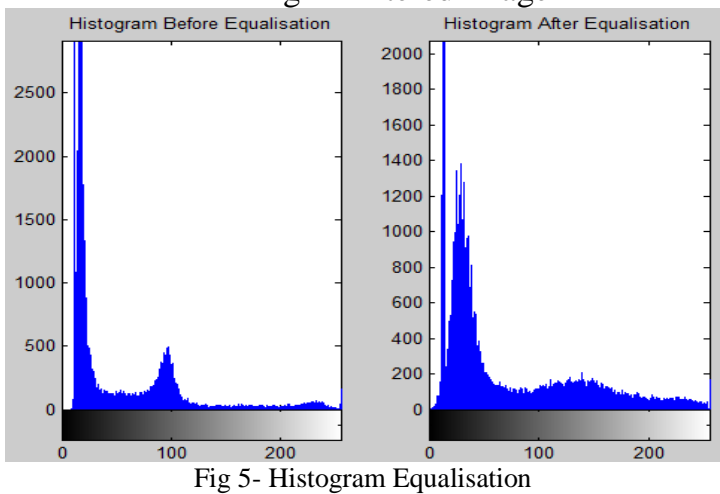

DOI 10.17148/IJARCCE.2015.45134
1) Textual Features:

Classification of image is done by textual features. So twelve features are extracted. These are given as below:

\begin{tabular}{|c|r|}
\hline & \multicolumn{1}{|c|}{ Value } \\
\hline Contrast & 0.6894 \\
\hline Correlation & 0.8939 \\
\hline ClusterProminence & 504.1630 \\
\hline ClusterShade & 49.0534 \\
\hline Dissimilarity & 0.3879 \\
\hline Energy & 0.1781 \\
\hline Entropy & 2.4959 \\
\hline Homogeneity & 0.8441 \\
\hline Homop & 0.8345 \\
\hline Max.Prob & 0.3229 \\
\hline Sosvh & 10.3088 \\
\hline Autocorrelation & 10.0477 \\
\hline
\end{tabular}

Fig 6 Texture Features

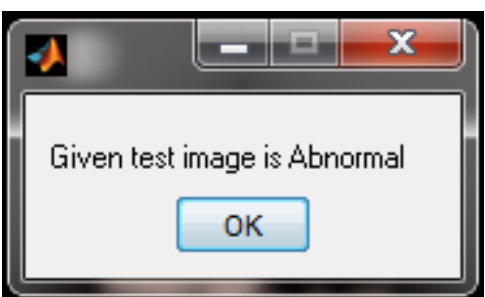

Fig 7- Decision of Classifier
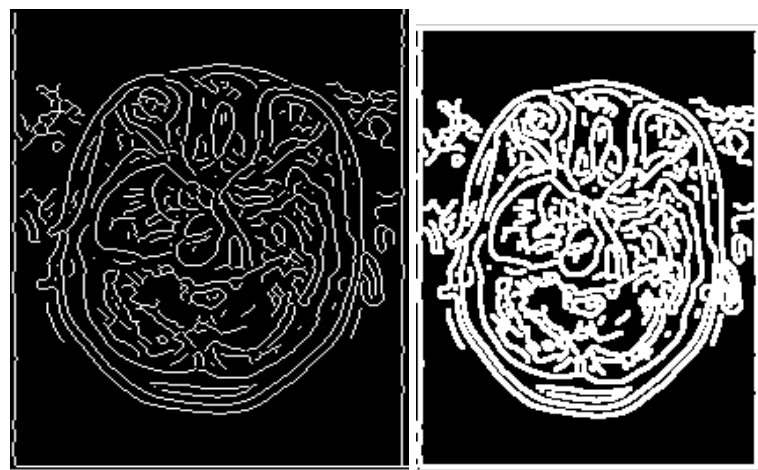

Fig 10 Binary \& Dilated Gradient Mask
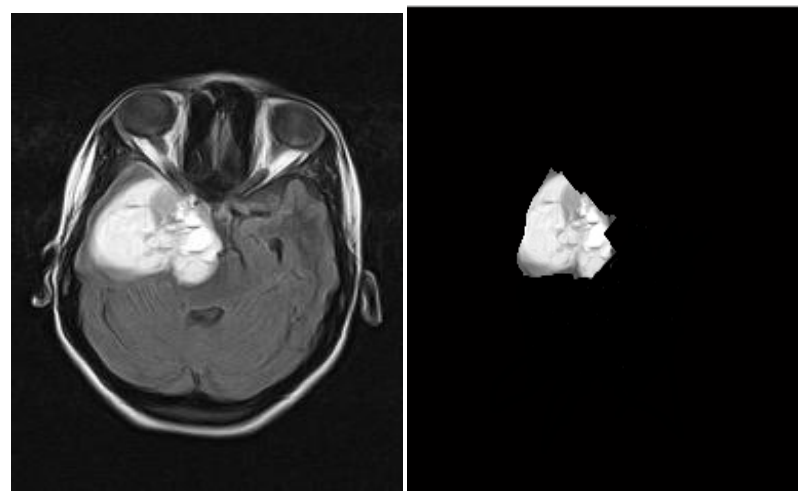

Fig 11 Threshold Segmented Image (White portion indicates tumour) 


\section{IV.CONCLUSION}

Image processing plays vital role in today's world. It has important application in biomedical field. The proposed system is developed for the diagnosing of tumour from magnetic resonance imaging pictures of the brain. This method makes the diagnosing in many phases. In the pre-processing stage filtering is performed on brain MR images. After that texture features are extracted from these noise free MRI images. These extracted features are used for classification. After classification into normal and timorous we detect tumour using edge detection \& basic morphology. This technique gives accurate and efficient results.

\section{ACKNOWLEDGMENT}

Kshitija Shingare would like to thank Prof. N.D. Pergad for their immense support and help.

\section{REFERENCES}

[1] Rohini Paul Joseph, C.Senthil Singh, M.Manikandan " Brain Tumor MRI Image Segmentation \& Detection In Image Processing", International Journal Of Research in Engineering \& Technology Vol.3, Issue 01, 2014.

[2] Jay Patel, Kaushal Doshi "A Study of Segmentation Methods for Detection of Tumor in Brain MRI", Adavance in Electronic\& Electrical Engineering, Vol.4.

[3] Roopali R.Laddha, S.A. Ladhake "A Review on Brain Tumor Detection Using Segmentation And Threshold Operations", International Journal of Computer Science \& Information Technologies", Vol.5, 2014.

[4] Prinyanka, Balwinder Singh "A Review On Brain Tumor Detection Using Segmentation", International Journal of Computer Science \& Mobile Computibg, Vol 2,Issue.7, 2013.

[5] Ravikumar M Sinojiya, Assi Prof Lokesh Gagnani, "An Image Segmentation to Detect Tumor and Measuring Size of Tumor Using Segmentation of MR Image", International Journal of Engineering Research and Application, Vol. 3, Issue 5, 2013.

[6] M.Karuna, Ankita Joshi "Automatic detection and Severityb Analysis of Brain Tumors using GUI in Matlab", International Journal of Research in Engineering and Technology, Vol.2, Issue 10, Oct 2013.

[7] Anam Mustaqeem, Ali Javed, Tehseen Fatima "An Efficient Brain Tumor Detection Algorithm Using Watershed \& Thresholding Based Segmentation", Image Graphics \& Signal Processing, 2012.

[8] Sarbani Datta, Dr. Monisha Chakraborty, "Brain Tumor Detection from Pre-Processed MR Images using Segmentation Techniques", IJCA Special Issue on $2^{\text {nd }}$ National Conference- Computing, Communication and Sensor Network, 2011.

[9] Swapnali Sawakare, Dimple Chaudhari, "Classification of Brain Tumor using Discrete Wavelet Transform, Principle Component Analysis \& Probabilistic Neural Network”, International Journal For Research In Emerging Science And Technology, Volume-1, Issue-6, November-2014.

[10] P.Sangeetha, "Brain Tumor Classification using PNN \& Clustering", International Journal of Innovative Research in Science, Engineering and Technology Volume 3, Special Issue 3, March 2014

[11] Shweta Jain "Brain Cancer Classification Using GLCM Based Feature Extraction in Artificial Neural Network", International Journal of Computer Science \& Engineering Technology Vol. 4, No. 07 Jul 2013

\section{BIOGRAPHY}

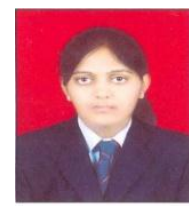

Kshitija V. Shingare was born on June 01, 1990. She is a PG Student in STB College of Engineering, Tuljapur (MH).

She received her B.E. in Electronics \& Telecommunication Engineering in 2012 and now pursuing masters in the same from BAMU Aurangabad University. Her research interests include image processing \& neural networking, in $2 \mathrm{D}$ and $3 \mathrm{D}$. 